\section{Quantification of Contaminant Removal \\ by Evactron Cleaning Using Quartz Crystal Thickness Monitors}

Christopher G. Morgan, Mark M. Gleason and Ronald Vane XEI Scientific, Inc.

Redwood City, CA

gabe@evactron.com

Hydrocarbon (HC) contamination is a persistent problem for users of electron microscopes (EMs), often leading to image distortion and interference with nanoprobing [1-4]. The Evactron De-Contaminator (D-C) has been available for HC contamination removal in EMs since 1999 [5]. The Evactron D-C uses low power radio frequency $(\mathrm{RF})$ generated plasma in order to produce oxygen radicals that clean the EM. The Oxygen Radical Source (ORS) is attached to the EM chamber, and a controlled leak of oxygen containing gas such as room air is passed through the plasma in order to produce oxygen radicals. The oxygen radicals chemically react with the HCs to form volatile oxidation products such as $\mathrm{H}_{2} \mathrm{O}, \mathrm{CO}$ and $\mathrm{CO}_{2}$. These volatile compounds are pumped out of the EM chamber.

The efficiency of the Evactron process can depend on several parameters; the pressure measured in the plasma, the power of the RF generator, the location in the chamber of the cleaning, and whether there are any obstacles between the plasma and the area to be cleaned. Quantifying the amount of HC contamination present and the rate of decontamination by easy, low cost methods has been a challenging problem. As of yet, no studies have been done to determine what effect these parameters have on the efficiency of the Evactron process.

Quartz crystal thickness monitors (QCTMs) are a standard technique for measuring the vacuum deposition of thin films and are available from many manufacturers [6]. They can also be adapted to measure HC contamination removal by remote plasma cleaning, and they are used to record a thickness loss rate of an oil layer previously deposited on their surface; this loss rate is a measure of the cleaning effectiveness of the Evactron D-C. This method provides a simple and inexpensive way to measure decontamination by the Evactron process, and it allows a determination of the effectiveness of the process changes as the operating parameters are allowed to vary.

The experimental set-up is shown in the picture in Figure 1. The size of the vacuum chamber used for the experiments is $30 \mathrm{~cm}$ diameter and $18 \mathrm{~cm}$ in height. It has four KF40 ports equidistant from each other. One of these ports is reserved for the vacuum pump; either an oil pump or a scroll pump was used in this system. The port opposite the vacuum line is reserved for the ORS. The third port has

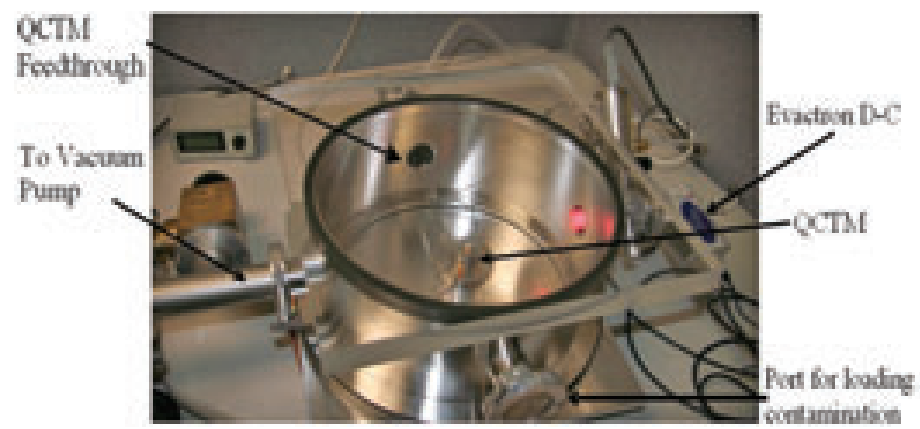

Figure 1: Picture of vacuum chamber used for QCTM studies with Evactron D-C. the electronic feedthroughs for the QCTMs, and the last for introducing contamination into the chamber. The signals from the QCTMs are monitored by a thickness monitor (McVac-MCM 160). Two QCTMs can be monitored with this device, and their output can be sent via an RS232 cable to a PC, where a LabVIEW ${ }^{\mathrm{m}}$ program can record the output as a function of time. A separate vacuum gauge can be used to monitor the pressure inside the chamber.

The following procedure is used to deposit HC contamination onto the QCTMs. Between 5-7 drops of liquid contamination (typically pump oil supplied by Duniway Stockroom Corp., part \# MPO190-1, but flaxseed oil is also used) are placed inside an $11 \mathrm{~cm}$ long vacuum tube. One end of the tube is attached to the chamber, and a leak valve is attached to the other end of the tube. The QCTMs are placed close to the port with the vacuum tube attached. The chamber is pumped down, and its pressure is adjusted to $\sim 0.15 \mathrm{mbar}$ using the leak valve. The vacuum tube is heated until a layer of oil $\sim 0.05$ $\mu \mathrm{m}$ is deposited on the QCTMs. A typical trace of thickness gain as a function of time on the QCTMs is shown in Figure 2. The tube is then allowed to cool, and the chamber is opened. Excess oil in the vacuum tube, on the walls of the chamber, and on the mounts for the QCTMs is removed using isopropyl alcohol wipes.

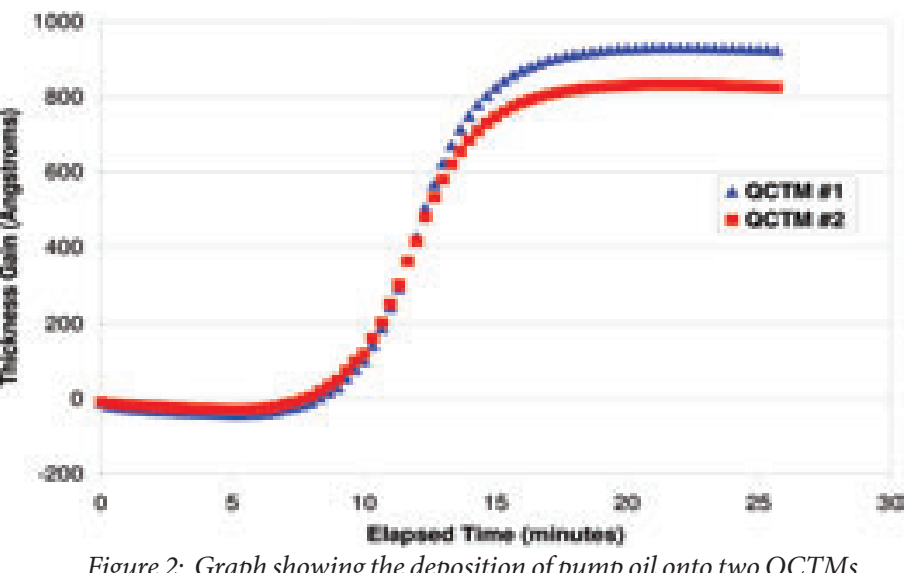

Figure 2: Graph showing the deposition of pump oil onto two QCTMs. See text for description of how the oil was deposited.

When the Evactron D-C is turned on shortly after a fresh layer of oil has been deposited, there is an initial increase in the thickness, as seen in Figure 3. When the Evactron process has started, the oil in the contamination layer begins to be oxidized by the radicals produced in the plasma. However, there is an induction period during the initial Evactron D-C activity. During this period, the oxygen radicals are incorporated into the oil layer, causing an increase in the thickness of the oil layer. Note also the delay between the minima in the signal for the QCTM in the center of the chamber and the minima in the signal for the QCTM on the side of the chamber, due to the longer time needed for the oxygen radicals to reach the QCTM on the side. Once enough oxygen radicals reach the surface and are incorporated into it, the oil layer can be turned into volatile compounds and removed. This removal, or thickness loss, occurs at a steady rate, as seen after 10-15 minutes elapsed time in Figure 3. The thickness loss rate is fairly repeatable under the same Evactron D-C operating conditions.

Using the method described above, the cleaning efficiency of the Evactron D-C as a function of chamber pressure and RF power can now be determined. The amount of air leaked into the chamber and the plasma was varied so that the pressure in the chamber was between 0.2 to 0.7 Torr, and the RF power was varied between 10, 


\section{DISCOVER \\ NEW HORIZONE IN \\ NANO GCALE IMAGING}

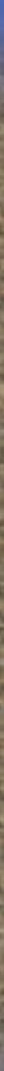

A new breed of microscope, powered by a unique helium ion beam, provides never-before seen, ultra-high resolution and material contrast.
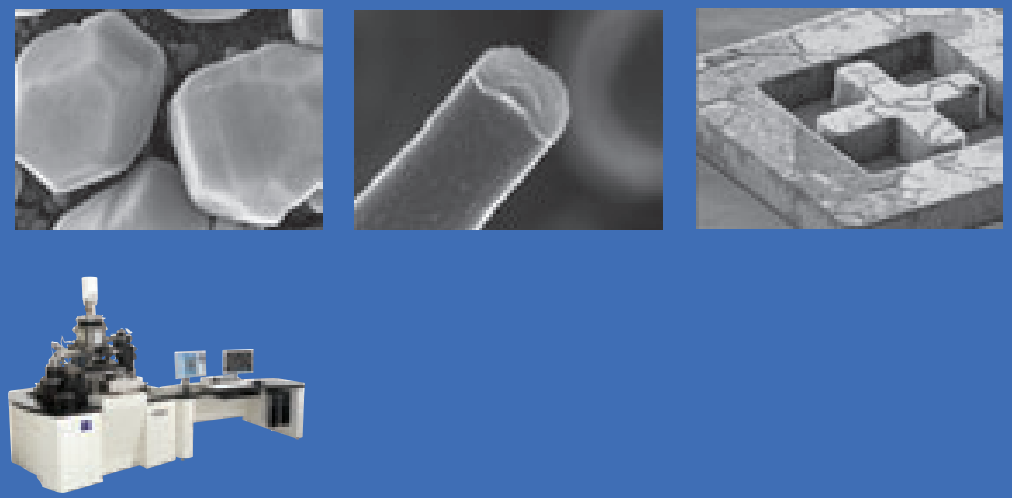

Carl Zeiss SMT Inc

One Corporation Way

Peabody, MA 01960

USA
Tel. +1 978/826 1500

Fax +1 978/532 2503

info-usa@smt.zeiss.com

www.smt.zeiss.com/nts

ZIISK 


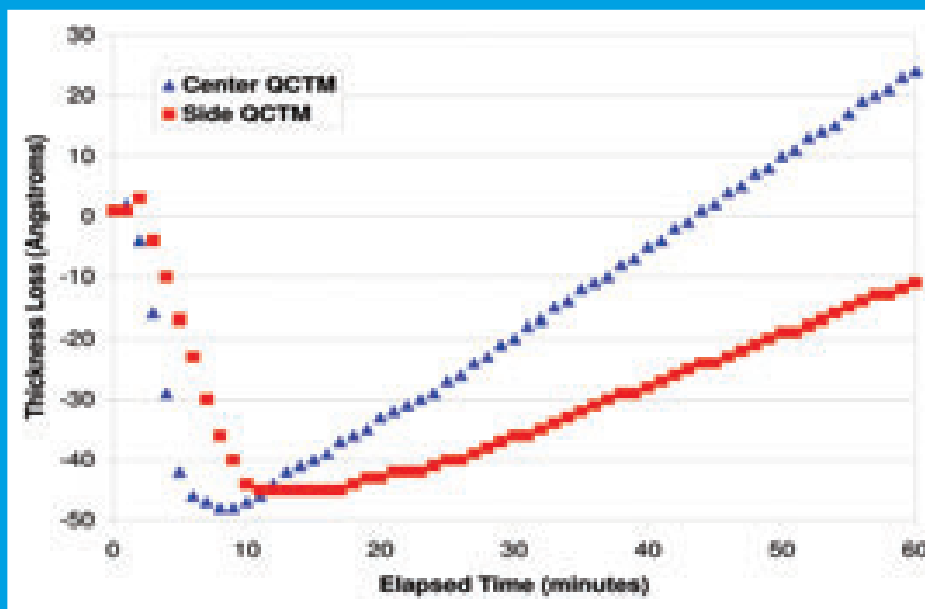

Figure 3: Initial activity seen during Evactron D-C cleaning immediately after depositing 0.065 um pump oil onto two QCTMs. The chamber pressure is set to 0.4 Torr and the RF power is set to $14 \mathrm{~W}$. Blue triangles ( $\mathbf{\Delta})$ show thickness loss of the QCTM placed in the center of the vacuum chamber, while red squares $(\mathbf{\square})$ show thickness loss of the QCTM placed on the side of the chamber. Note the induction period for both traces, during which the thickness of the contamination layer increases due to incorporation of oxygen radicals into the layer.

14 and $17 \mathrm{~W}$. The results are shown in Figure 4. As the pressure increases, the rate of thickness loss decreases. This decrease is due to the higher rate of three-body reactions in the plasma and chamber. These reactions destroy the oxygen radicals that decontaminate the chamber. It was expected that at very low pressures the efficiency will drop again, due to the lack of oxygen radicals being produced, but this turning point was not reached by our experiments. This may be because either the three body recombination rate at low pressures is so small that relatively large numbers of oxygen radicals make it into the chamber. Alternatively, other processes related to the plasma, such as ions or highly energized species, can reach further into the chamber and affect the QCTM thickness loss rate.

As expected for the power variation, an increase in power generally leads to an increase in decontamination efficiency. The increase in decontamination efficiency between 14 and $17 \mathrm{~W} \mathrm{RF}$ power is not as great as the corresponding increase between 10 and $14 \mathrm{~W}$. Although more oxygen radicals are produced when the RF power is increased, the number of $\mathrm{N}_{2}{ }^{+}$ions in the plasma also increases.

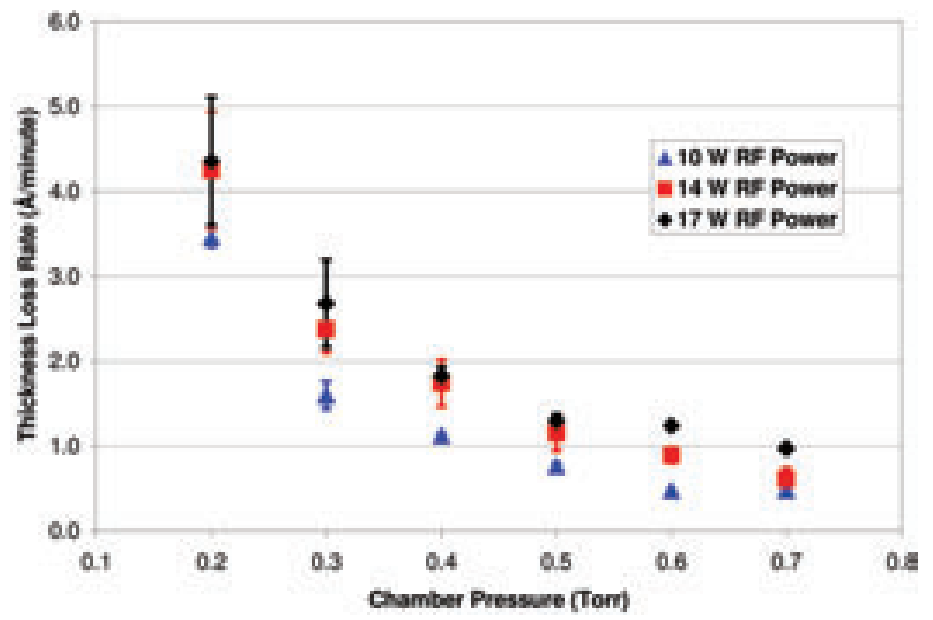

Figure 4: Variation in thickness loss rates as a function of chamber pressure and RF power. Data was taken from a QCTM placed in the center of the chamber. Blue triangles ( $\mathbf{\Delta})$ show thickness loss rates when the Evactron $D-C$ is at $10 \mathrm{~W} R F$ power. Red squares ( $\mathbf{\square})$ show thickness loss rates at $14 \mathrm{~W} R F$ power, and black diamonds $(\bullet)$ show thickness loss rates at $17 \mathrm{~W} R \mathrm{RF}$ power.
These ions destroy oxygen radicals, and decrease the total number of oxygen radicals produced.

In addition, a study was done to see what effect the distance between the ORS and the thickness meter has on the cleaning efficiency; the results are shown in Figure 5. These experiments were done with flaxseed oil loaded onto the thickness monitor, with the RF power at $14 \mathrm{~W}$, and with the chamber pressure at different levels. A single QCTM was placed on an imaginary line between the ORS port and the vacuum port. The efficiency of the Evactron process increased, and the pressure dependence was greater the closer the thickness meter was to the ORS. These changes can also be explained by the number of oxygen radical destroying three-body reactions occurring. The higher the chamber pressure and the longer the oxygen radicals travel before they react on the thickness monitor, the more likely they will be destroyed.

This method can also be used to determine the shielding effects of collimators. These collimators are often found covering EDS windows. Previous studies have shown that up to 300 hours of Evactron cleaning has little effect on these windows [7].

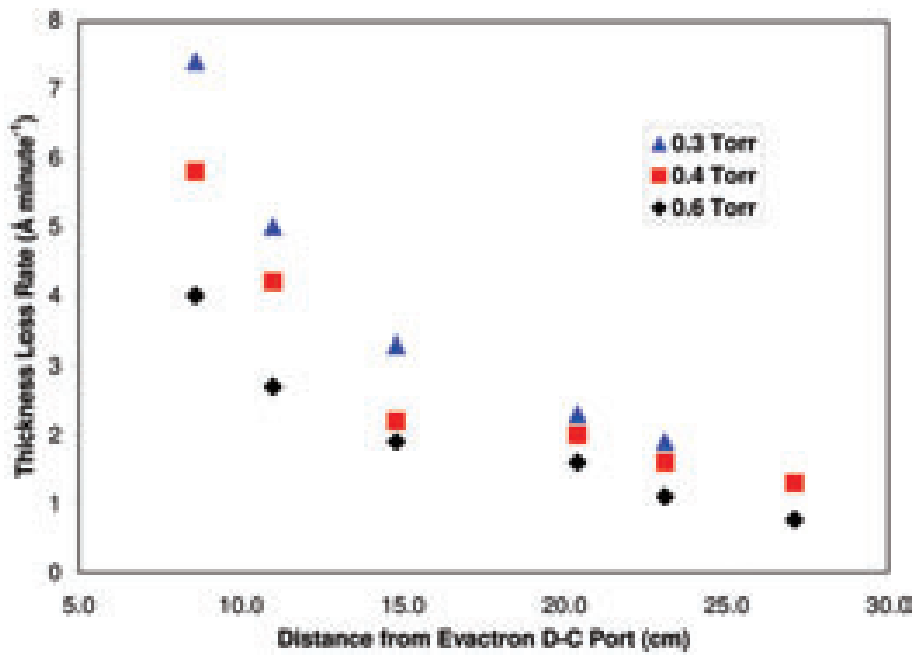

Figure 5: Variation in thickness loss rates as a function of distance between ORS and vacuum port. A single QCTM was placed on an imaginary line between the ORS and the vacuum port. The RF power was set at $14 \mathrm{~W}$. Blue triangles ( $\mathbf{\Delta})$ show thickness loss rates when the chamber pressure was at 0.3 Torr. Red squares ( $\mathbf{\square}$ ) show thickness loss rates when the chamber pressure was at 0.4 Torr, and black diamonds ( $)$ show thickness loss rates when the chamber pressure was at 0.6 Torr.

The collimators were provided by Oxford Instruments. It is in the shape of a tapered tube, with a large opening $(1.5 \mathrm{~cm})$ on the bottom and a small opening $(0.4 \mathrm{~cm})$ on the top. It is $2.5 \mathrm{~cm}$ in height. An insert was fabricated to reduce the diameter of the top opening. When the insert is in place, the diameter of the collimator is reduced from $4 \mathrm{~mm}$ to $1 \mathrm{~mm}$. The collimator was placed over one of the QCTMs; the other QCTM was left uncovered as a reference. A piece of Teflon tape was placed around the base of the collimator so that the only opening between the QCTM and the rest of the system was the opening at the top of the collimator. The quartz crystal thin film thickness meter and the collimators are shown in Figure 6.

The first set of experiments with collimator shielding was done without the $1 \mathrm{~mm}$ insert; the diameter of the collimator is $4 \mathrm{~mm}$. The results of this experiment are shown in Figure 7. In this graph, thickness loss (in Ångstroms) is shown as a positive number. When the Evactron process is turned off, almost no thickness loss is seen on either QCTM. The rate for the covered QCTM is about $4 \times$ less 


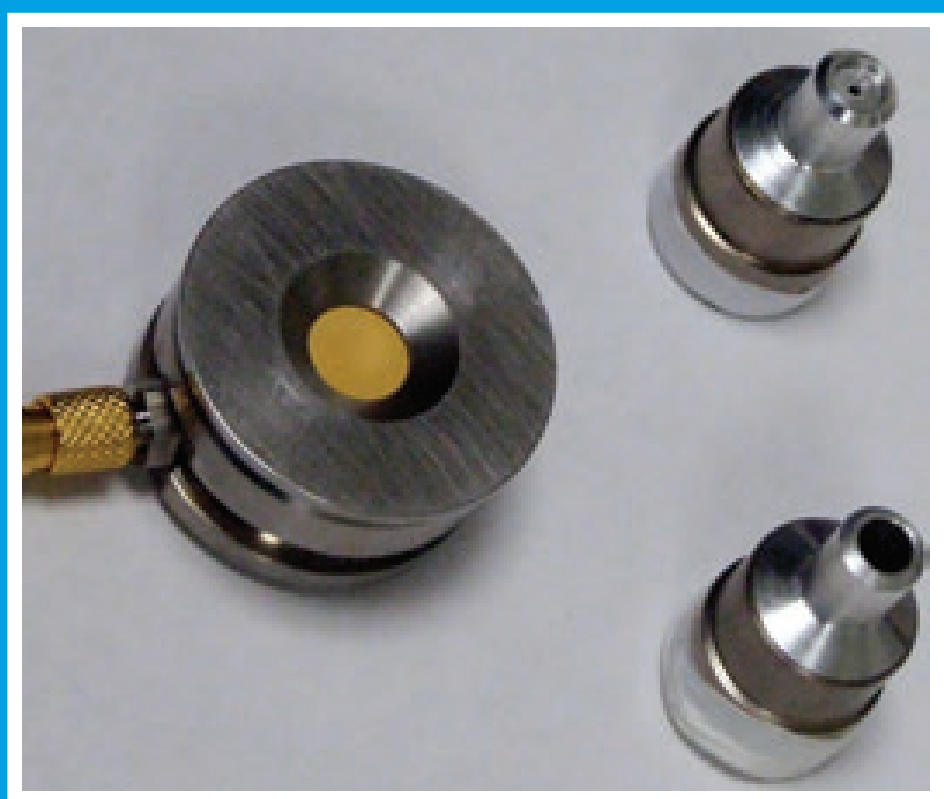

Figure 6: Quartz crystal thickness meter (left) and Oxford Instruments collimator with (top right) and without insert (bottom right). Teflon tape has been placed around the base of the collimators.

than the uncovered QCTM, which is the difference in area between the size of the quartz crystal QCTM and the size of the collimator opening. Oxygen radicals are able to diffuse through the collimator and remove contamination from the QCTM with minimal loss on the walls of the collimator. The collimator is made from aluminum, and the native aluminum oxide surface will not remove oxygen radicals very efficiently. The rate of thickness loss is determined by the geometry of the experiment.

This experiment was repeated at a lower pressure to see what effect a change in the Knudsen number will have on the ratio between the uncovered and covered thickness loss rates. The ratio between the uncovered and covered rates doubles when the chamber pressure is halved, suggesting that the Knudsen number can be used to ascertain the shielding effect of the collimator.

The second experiment with collimator shielding has the $1 \mathrm{~mm}$ insert placed in the collimator. The results of this experiment are shown in Figure 8. This time, however, there is a radical difference between thickness loss rates. For the uncovered QCTM, the thickness loss rate was measured to be $1.9 \AA /$ minute. Using the geometric

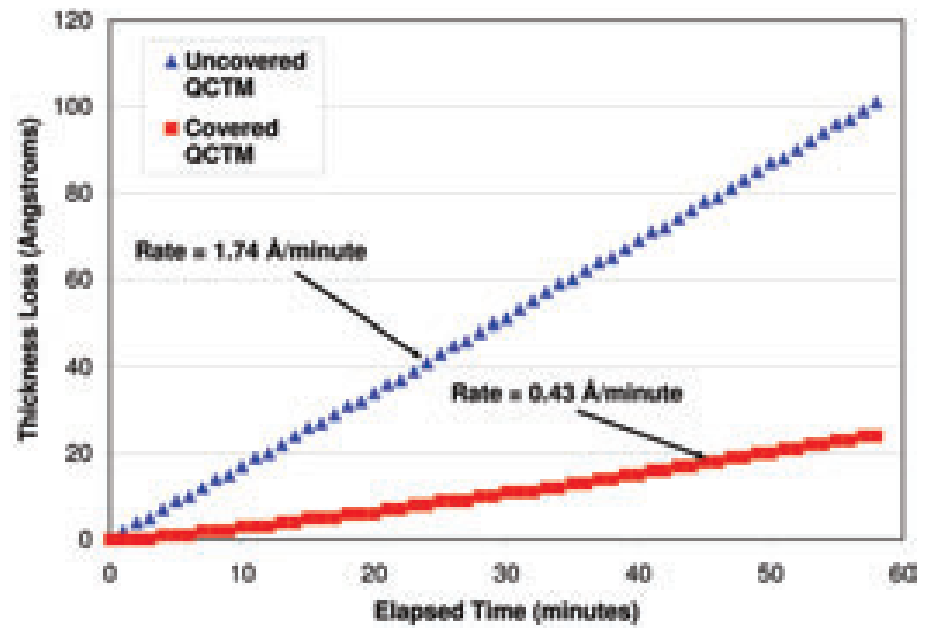

Figure 7: Comparison of thickness loss rates between an uncovered QCTM, shown as blue triangles ( $\mathbf{\Delta})$, and a QCTM covered by a collimator with a $4 \mathrm{~mm}$ diameter, shown as red squares ( $\mathbf{\square})$. The chamber pressure was set to 0.4 Torr, and the RF power of the Evactron D-C was set to $15 \mathrm{~W}$. arguments from the results of the first experiments, one would expect the loss rate of the covered QCTM to be around $0.2 \AA$ /minute and the total thickness loss after 80 minutes to be around 16 Angstroms. However, the rate of thickness loss for the covered QCTM and the 1 mm insert was actually around $0.04 \AA /$ minute. There is a change in the Knudsen number of the oxygen radicals entering the top of the collimator. When the collimator opening is reduced from $4 \mathrm{~mm}$ to 1 $\mathrm{mm}$, the Knudsen number increases 0.12 , further into the transition region between viscous and molecular flow [8]. It may be more difficult for the oxygen radicals to move through the top of the collimator if they are in a flow regime that is more molecular than viscous.

To conclude, the cleaning efficiency of the Evactron process was quantified by using quartz crystal thin film thickness meters. Chamber pressure, RF power, and distance between the ORS and the thickness meter were the parameters used in this study. Lower pressure and higher power leads to greater cleaning efficiency. This efficiency is less the further away from the ORS the area to be cleaning is located. The results indicate that three body collisions play an important role in determining the cleaning efficiency of the oxygen radicals, although there might be chemistry due to species created directly in the plasma, such as high energy radicals or ions, at low pressure.

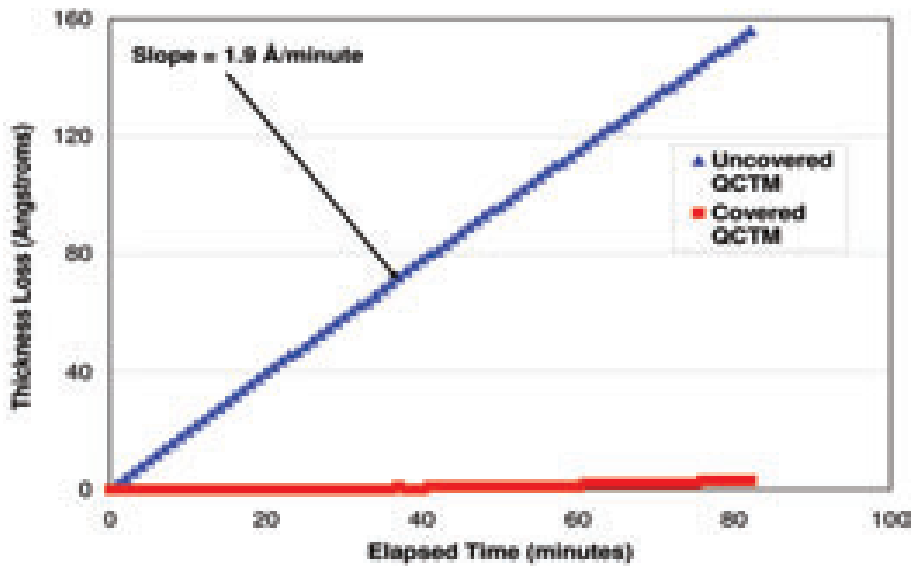

Figure 8: Comparison of thickness loss rates between an uncovered QCTM, shown as blue triangles ( $\mathbf{\Delta})$, and a QCTM covered by a collimator with the $1 \mathrm{~mm}$ diameter insert, shown as red squares ( $\square)$. The chamber pressure was set to 0.4 Torr, and the RF power of the Evactron D-C was set to $15 \mathrm{~W}$.

The results of the collimator study suggest that an increase in the Knudsen number will decrease the ability of the oxygen radicals to get through the collimator. The Knudsen number can be increased by lowering the pressure and making the collimator diameter smaller. The collimator shields the EDS windows from the oxygen radicals produced by the Evactron D-C, and this shielding extends the expected time that the Evactron process can be safely run with these windows in the chamber.

\section{References}

[1] A. Vladár, M. T. Postek, and R. Vane, Contamination. Proc. SPIE (2001) 4344:835-843

[2] M. T. Postek, SCANNING (1996) 18:269-274

[3] N. Sullivan, T. Mai, S. Bowdoin and R. Vane, Microsc. Microanal. 8 (Suppl 2) (2002) $720 \mathrm{CD}$

[4] L. Reimer, SPIE Opt. Eng. Press, Volume TT 12 (1993)

[5] http://www.evactron.com/index.html

[6] Milton Ohring, The Materials Science of Thin Films, 2nd edition, Academic Press, 2001

[7] R. Vane, C. Roberts, and V Carlino, Microsc. Microanal. 10 (Suppl 2) (2004) 966CD

[8] A User's Guide to Vacuum Technology, $2^{\text {nd }}$ Ed., John O'Hanlon, John Wiley \& Sons, 1989. 